\title{
BMJ Global Health Lactational amenorrhoea among adolescent girls in low-income and middle-income countries: a systematic scoping review
}

\author{
Martines N S Figaroa, ${ }^{1}$ Saverio Bellizzi, ${ }^{2}$ Therese Delvaux, ${ }^{3}$ Lenka Benova ${ }^{3}$
}

\begin{abstract}
To cite: Figaroa MNS,
Bellizzi S, Delvaux T, et al. Lactational amenorrhoea among adolescent girls in lowincome and middle-income countries: a systematic scoping review. BMJ Global Health 2020;5:e002492. doi:10.1136/ bmjgh-2020-002492
\end{abstract}

Handling editor Kerry Scott

- Additional material is published online only. To view, please visit the journal online (http://dx.doi.org/10.1136/ bmjgh-2020-002492).

Received 16 March 2020 Revised 31 August 2020 Accepted 1 September 2020

Check for updates

(C) Author(s) (or their employer(s)) 2020. Re-use permitted under CC BY-NC. No commercial re-use. See rights and permissions. Published by BMJ.

${ }^{1}$ Department of Epidemiology and Social Medicine, University of Antwerp, Antwerpen, Belgium ${ }^{2}$ Partnership for Maternal, Newborn and Child Health, Geneva, Switzerland

${ }^{3}$ Department of Public Health, Institute of Tropical Medicine, Antwerpen, Belgium

Correspondence to Dr Lenka Benova; Ibenova@itg.be

\section{ABSTRACT}

Introduction Fertility levels among adolescents remain high in many settings. The objective of this paper was to review the available literature about postpartum and lactational amenorrhoea among adolescents in low-income and middle-income countries (LMICs).

Methods We searched Medline, Embase, Global Health and CINAHL Plus databases using terms capturing adolescence and lactational or postpartum amenorrhoea. Inclusion criteria included publication date since 1990, data from LMICs, and topic related to lactational amenorrhoea as a postpartum family planning method or as an effect of (exclusive) breast feeding among adolescents. Thematic analysis and narrative synthesis were applied to summarise and interpret the findings. Results We screened 982 titles and abstracts, reviewed 75 full-text articles and included nine. Eight studies assessed data from a single country (three from India, two from Bangladesh, two from Turkey, one from Nigeria). One study using Demographic and Health Survey data included 37 different LMICs. The five studies measuring duration of postpartum or lactational amenorrhoea reported a wide range of durations across the contexts examined. Four studies (from Bangladesh, Nigeria and Turkey) examined outcomes related to the use of lactational amenorrhoea as a family planning method among adolescents. We did not find any studies assessing adolescents' knowledge of lactational amenorrhoea as a postpartum family planning method. Likewise, little is known about the effectiveness of lactational amenorrhoea method among adolescents using sufficiently large samples and follow-up time.

Conclusion The available evidence on lactational amenorrhoea among adolescents in LMICs is scarce. Given the potential contribution of lactational amenorrhoea to prevention of short interpregnancy intervals among adolescents and young women, there is a need for a better understanding of the duration of lactational amenorrhoea, and the knowledge and effective use of lactational amenorrhoea method for family planning among adolescents in a wider range of LMIC settings.

\section{INTRODUCTION}

Early pregnancy and parenthood are some of the most prominent challenges with which

\section{Key questions}

\section{What is already known?}

- Lactational amenorrhoea method (LAM) is an effective postpartum contraceptive method available to breastfeeding women and does not require a health provider or replenishment of contraceptive supplies.

- The duration of lactational amenorrhoea and the role of LAM as a family planning method among adolescents in low-income and middle-income countries (LMICs) are not known.

\section{What are the new findings?}

- We identified nine studies from LMICs, all of which were quantitative and observational.

- There was heterogeneity in the findings about the duration of lactational amenorrhoea among adolescents compared with older women across these settings.

- We identified evidence gaps surrounding adolescents' knowledge of LAM and transition from the use of LAM to other contraceptive methods.

\section{What do the new findings imply?}

- This study highlights the need for a better understanding of breastfeeding practices, barriers and enablers of LAM use among adolescents.

- There is a need for additional research in a wider range of settings and using qualitative research methods.

adolescents globally are dealing. Approximately 16 million girls aged 15 to 19 years and 2.5 million girls under 16 years give birth each year in low-income and middle-income countries (LMICs). ${ }^{1-3}$ Despite the global decline in adolescent birth rate between 1990 (64.8 births per 1000 girls $15-19$ years of age) and 2020 (42.5 births per 1000), ${ }^{4}$ the number of adolescent pregnancies globally will continue to increase due to the size of the adolescent cohorts, with the greatest proportional increase in West and Central Africa and Eastern and Southern Africa. ${ }^{5}$ 
It is estimated that in 2020,257 million women globally had an unmet need for modern contraception, and 218 million of them were in developing countries. ${ }^{6}$ Accessibility and availability of contraceptives for adolescent girls, especially unmarried, in LMICs are even more problematic compared with older women of reproductive age. ${ }^{7}$ Different barriers including stigma, social pressure, legal restrictions, provider biases and misinformation may prevent adolescents from obtaining contraceptives. ${ }^{8} 9$ Additional barriers include interruptions in contraceptive supplies and lack of financial affordability. ${ }^{10}$ A study estimated that $90 \%$ of the over 6 million annual unplanned pregnancies, either unwanted or mistimed, among adolescent girls in Sub-Saharan Africa, Latin America and the Caribbean, and South Central and Southeast Asia are due to non-use of a modern method of contraception. ${ }^{11}$ In 2016, an estimated 38 million adolescents in developing regions wanted to avoid pregnancy, 23 million of them have an unmet need for modern contraception and are thus at elevated risk of unintended pregnancy. ${ }^{11}$

Nearly one-fifth of young women in LMICs are estimated to become pregnant before age 18 , and 2 million births occur to girls under age 15 annually in LMICs. ${ }^{12}$ For example, median age at first childbirth among women 20-24 years was $<20$ years in all 16 Sub-Saharan African countries with a Demographic and Health Survey collected since 2010 and where this indicator is available. ${ }^{13}$ While not all adolescent pregnancies are unintended, almost half of the 20 million pregnancies among adolescents in LMICs are. ${ }^{14}$ Pregnancy and childbirth complications are the leading cause of death among girls 15 to 19 years old globally. ${ }^{15-17}$ Compared with babies of women in their twenties, infants born to adolescents face a higher risk of preterm birth, which is among the leading causes of neonatal mortality and morbidity. ${ }^{18}$ First-order births carry an increased risk of complications, ${ }^{19}{ }^{20}$ and in many LMICs, the majority of first-order births are to adolescent girls. $^{21}$

Existing limited research from LMICs shows that repeat teenage pregnancy or childbirth is common. ${ }^{22}{ }^{23} \mathrm{New}$ evidence is emerging on the length of what constitutes a short interpregnancy interval and its effects on maternal, perinatal and child survival and health outcomes. ${ }^{24}{ }^{25} \mathrm{In}$ LMICs, short interpregnancy intervals and other factors appear to play an important role in an increased risk of adverse outcomes among adolescent mothers with repeat pregnancies and their babies. ${ }^{26-28}$ The concept of an ideal interpregnancy interval emerged from a report published by WHO in 2005 and, based on the best available evidence at that time, consensus was reached that an optimal interval was a minimum of 24 months, ${ }^{29}$ consistent with the joint WHO and Unicef recommendation for women to breast feed for at least 2 years. ${ }^{30}$

Immediately following childbirth, the inhibitory effect of oestrogen and progesterone levels of pregnancy decreases, with the resumption of regular ovulation at around 25 days after birth. ${ }^{24}$ Consequently, all postpartum women are assumed to be protected from conception for 4 weeks following childbirth. The period of postpartum amenorrhoea can be prolonged by breast feeding (lactational amenorrhoea), which changes the level and rhythm of gonadotropin-releasing hormone $(\mathrm{GnRH})$ secretion by sending neural signals to the mother's hypothalamus through stimulation of the nipple. GnRH influences the pituitary release of follicle-stimulating hormone and luteinising hormone, the hormones needed to stimulate and resume ovulation. ${ }^{31}$

Lactational amenorrhoea method (LAM) is the name given to the informed use of breast feeding as a method of contraception. For lactational amenorrhoea to serve as an effective method of contraception, the woman must be exclusively or near exclusively breast feeding (at least $85 \%$ of infant feeding coming from breast feeding), ${ }^{29}$ be within the first 6 months following childbirth, and remain amenorrhoeic. The typical use failure rate of LAM is $0.45 \%-7.5 \% .^{32}$ In the first 6 months post partum, amenorrhoeic women have a very low cumulative chance of conception, even if they are not exclusively breast feeding, because a large fraction of first menstrual cycles in this period are anovulatory. ${ }^{33}$ As the duration of post partum lengthens, the protective effect of amenorrhoea progressively weakens. Nevertheless, among amenorrhoeic women, the level of risk of conception remains at $6 \%$ at 12 months post partum, ${ }^{34}$ which is not substantively different from that of condoms or oral contraception under real-life conditions. Literature has shown that reliance on the absence of menses as an indicator that conception is unlikely is widespread. ${ }^{35}$ However, most women do not associate breast feeding with a reduced risk of conception. ${ }^{35}$ Beyond 6 months post partum, women might continue breast feeding and remain amenorrhoeic. While this period is included in calculations of the duration of lactational amenorrhoea, it is no longer considered to be an effective contraceptive method.

Addressing the unmet need for family planning is of paramount importance to improve the lives of girls and young women, particularly in LMICs. Despite the significant implications that countries face if this issue remains neglected, adolescents' sexual and reproductive health has traditionally been overlooked. LAM is available to breastfeeding women, does not require a health provider or replenishment of contraceptive supplies, and is effective at preventing pregnancy (it is classified as a modern contraceptive method). ${ }^{36} 37$ Therefore, it can play a role in efforts to address unintended repeat childbearing among adolescents, including pregnancies preceded by a short interpregnancy interval. However, little is known about the extent to which adolescents in LMICs know about this method, are aware of the criteria for its effective use and are using it. If adolescent girls face different barriers, a targeted approach to awareness-raising about LAM might be required in contrast to mothers from older age groups. In order to understand these issues better, a thorough search in the literature can help map current evidence. This scoping review was conducted to 
answer the following primary research question: What is the current state of evidence on knowledge about postpartum/lactational amenorrhoea among adolescents in LMICs?

\section{Objective}

The objective of this review is to systematically scope the published literature, to synthesise what is known about postpartum/lactational amenorrhoea among adolescents in LMICs and to identify existing gaps in available evidence.

\section{METHODS}

\section{Search strategy}

Our review was guided by the standard principles of Arksey and O'Malley's framework and the PRISMA-ScR checklist (online supplemental material 1). ${ }^{38}{ }^{39}$ Arksey and O'Malley's approach can be described as an iterative process involving post hoc inclusion and exclusion criteria. According to this framework, there are five stages: (1) identifying the research question, (2) identifying relevant studies, (3) study selection, (4) charting the data and lastly (5) collating, summarising and reporting the results. The optional step of consultation exercise involving key stakeholders to validate findings was not found necessary in this study and was not performed. The protocol for this scoping review was not registered.

We searched four databases, Medline, Embase, Global Health and CINAHL Plus, using a combination of search terms comprising the terms adolescents and lactational amenorrhoea (full electronic search strategy is presented in online supplemental material 2). After deduplication, titles and abstracts of identified references were all screened independently by two reviewers (MNSF and LB). Additional references were identified through hand searching the DHS programme publications site, ${ }^{40}$ the website of the Population Council, ${ }^{41}$ the WHO Reproductive Health Library ${ }^{42}$ and reference lists of all articles reviewed in full text.

\section{Eligibility criteria}

We applied the following inclusion criteria during title/abstract and full-text search: (1) studies published between 1990 (included) and 23 September 2019 (date of the search), because only after August 1988 consensus on LAM was reached through the Bellagio consensus ${ }^{43}$; (2) contained data collected in LMIC as defined by the World Bank ${ }^{44}$; (3) can be a research paper (qualitative or quantitative), editorial or commentary, peer-reviewed paper or not (report, research paper), but conference abstracts were included only if they presented research results; (4) data were presented (or disaggregated) for adolescent girls between the age of 10 and 19 years; (6) the topic of lactational amenorrhoea was examined as a postpartum family planning method, or as an effect of (exclusive) breast feeding, including through quantitative indicators such as median duration or knowledge of lactational amenorrhoea, or qualitative analysis such as perceptions or barriers to use.

Studies were excluded if they (1) mentioned amenorrhoea in a context without previous childbirth/ pregnancy (eg, amenorrhoea among girls treated for

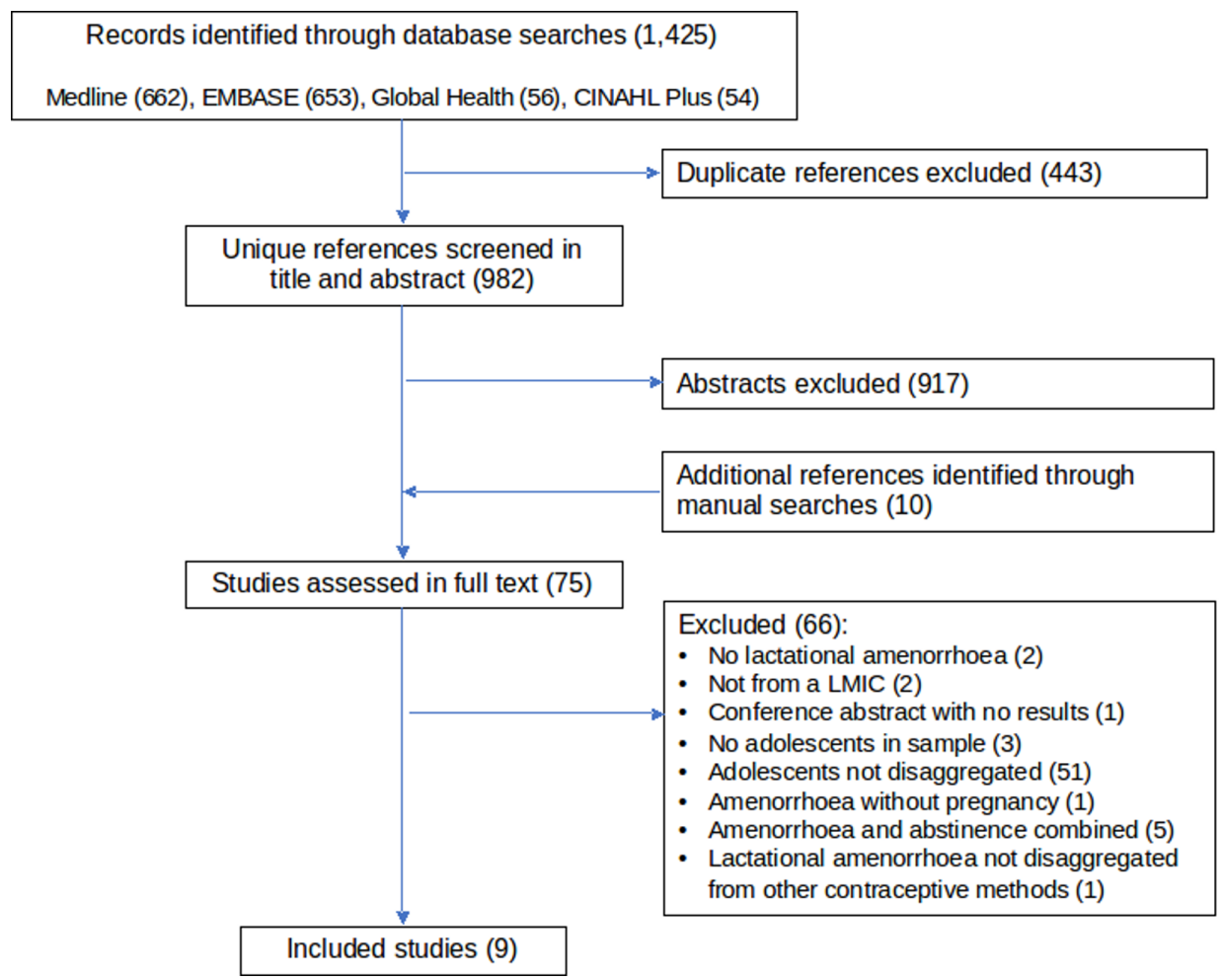

Figure 1 Scoping review search flowchart. 
anorexia or following cancer treatment, primary amenorrhoea among adolescents), (2) presented measures of postpartum infecundability (amenorrhoea and abstinence combined, without disaggregating lactational amenorrhoea separately), and (3) examined contraceptive/family planning use and lactational amenorrhoea combined with other family planning methods without disaggregation. Two reviewers (MNSF and LB) independently screened all full-text articles. Differences were reconciled through discussion and consensus. Figure 1 presents the full search flowchart.

\section{Data charting process}

To extract relevant data from the references included in full text, a standard template sheet was used specifying the author(s), year of publication, journal, time of data collection, country(ies), site within country(ies), objective of each study, study design, sample size of adolescents, description of the sample, recruitment and eligibility, follow-up period (prospective studies) or time since childbirth (retrospective studies), completeness of follow-up or missingness of data, measurement/analysis method, definition(s) of the lactational amenorrhoea outcome(s) or indicator(s) used, and key findings in reference to adolescents. Two coauthors (MNSF and LB) independently extracted all data from studies included in full text. Any differences were resolved through discussion. As is common for scoping reviews, we did not formally assess the quality of included studies.

\section{Collating, summarising and reporting the results}

Descriptive information about the included studies is summarised in a table. To synthesise and interpret the findings of this scoping review, we used thematic analysis and narrative synthesis. We summarised the methods used by included studies and report the findings of studies according to the key theme identified. Based on these findings, we highlighted the gaps in available literature.

\section{Patient and public involvement}

No patient or public involvement took place in the design or conduct of this literature review. The results are intended for wide dissemination, including to researchers, programme implementers and governmental agencies, all of whom reach the public and the key population of this study.

\section{RESULTS}

\section{Overview of included studies}

We screened 982 unique references in title and abstract, 75 in full text, and included nine in this review (figure 1). The main reason for exclusion of studies in full-text screening (51 out of 66 studies) was that while adolescents were included in the analysis sample, they were not disaggregated from women older than 20 years (at all or were included in a broader age category conflating adolescents and young women, eg, an age group from 15 to 24 years). Table 1 shows the characteristics of the included studies. Three studies were published in the 1990s, three in the 2000s, and three since 2010. Eight studies assessed data from a single country (three from India, two from Bangladesh, two from Turkey and one from Nigeria), and one study using Demographic and Health Survey data included 37 different LMICs (18 countries in SubSaharan Africa, 4 near East/North Africa, 7 in Asia, and 8 in Latin America and the Caribbean). One study from Uttar Pradesh in India ${ }^{45}$ appears to have used the same data as a second included study ${ }^{46}$; the results reported are identical.

Two of the nine included studies had as their main objective to investigate lactational amenorrhoea among adolescents. ${ }^{478}$ Three additional studies were interested in differentials in durations of amenorrhoea according to mother's age ${ }^{46}$ demographic and biodemographic characteristics, ${ }^{43}$ or were focused on sociodemographic influences. ${ }^{47}$ The remaining four studies did not specifically set out to investigate lactational amenorrhoea among adolescents or the effect of age on lactational amenorrhoea, but presented findings relevant to this scoping review. All nine included studies were quantitative and used observational study designs (eight used retrospective and one prospective data collection). One of the nine included studies had only adolescents in their sample; all other studies included women older than 20 years and provided comparisons with adolescents. Five studies were analytical, three of which reported findings from crude analysis ${ }^{49} 40$ and two conducted multivariable analyses of the association between age and lactational amenorrhoea. ${ }^{461}$ The remaining four studies presented descriptive analyses only, meaning they did not perform statistical tests of the differences between indicators of lactational amenorrhoea among adolescents and older women.

The studies included and disaggregated lactational amenorrhoea among females under the age of 20 (ie, adolescents). Five of the studies only included currently married and one study only ever-married women and girls. The remaining three studies did not specify any inclusion criteria or sample characteristics related to marital status. The age group relevant for this scoping review was defined as $<20$ years in four studies, 10-19 years in one study and 15-19 years in two studies, and 2 studies used further disaggregation by age among adolescents $(10-14$ and $15-19 ;<15$ and 15-20). Maternal age was measured at the time of birth of the index child (three studies), at the time of marriage (one study), at time of receiving antenatal care (one study), and at the time of survey or at some point during the study (not further specified) in the remaining four studies. To establish women's age, two studies (both from Turkey) used medical records to identify women eligible for their sample and might have collected the birth date or age of study participants from this source (it is unclear whether this was further validated when interacting with the respondents). The 


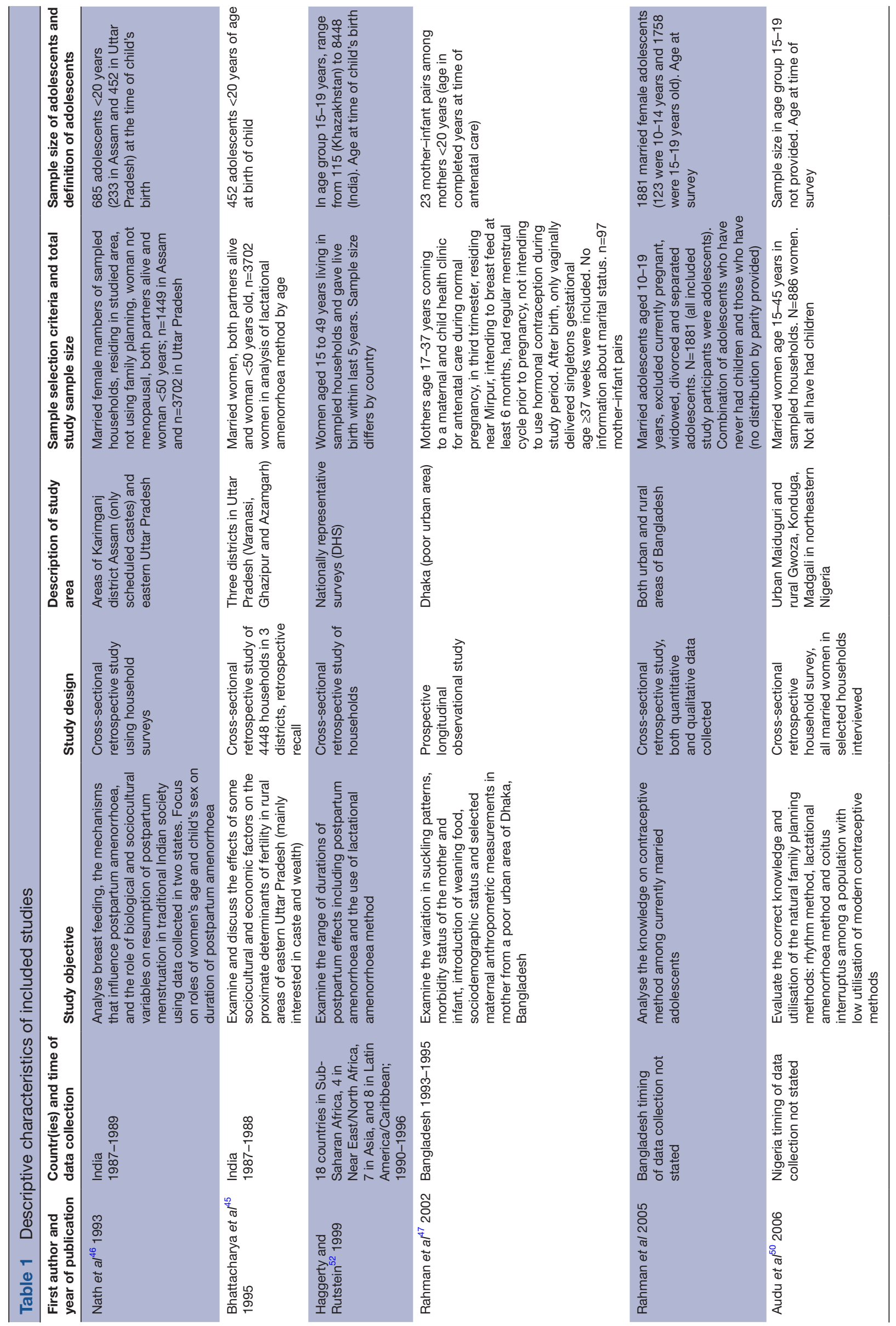




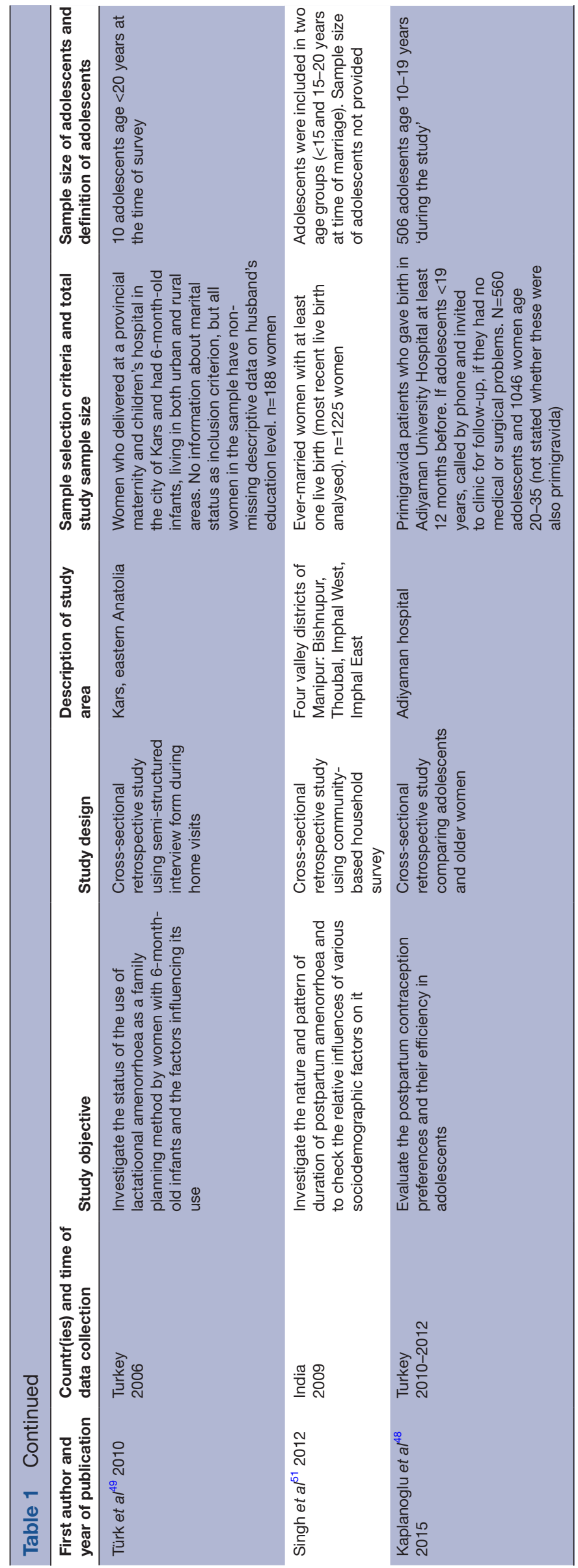

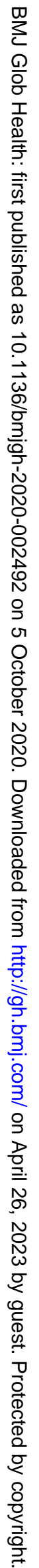


remaining seven studies relied on either a household member or the woman's own report of her age.

Table 2 summarises the nine studies' findings. We identified two key themes examined by included studies. The first theme, identified in five studies, captures the length of amenorrhoea. This was expressed either as median duration in months or weeks and/or the percentages of women in the sample resuming menses (or the opposite, remaining amenorrhoeic) at specific time intervals since the birth. The second theme concerned the use of lactational amenorrhoea as a family planning method and was used by four studies. No study presented findings related to both themes. All nine studies relied on respondent's recall to capture data relevant to the length of lactational amenorrhoea, the use of lactational amenorrhoea as a family planning method or return of menses. None of the studies used biomarkers to measure or validate selfreported outcomes.

\section{Theme 1: duration and outcomes of postpartum or lactational amenorrhoea}

The five studies measuring duration of postpartum or lactational amenorrhoea reported a wide range of durations across the contexts examined. In Assam ${ }^{46}$ and Bangladesh, ${ }^{47}$ duration of lactational amenorrhoea did not appear to differ between adolescents and women older than 20 years. In Uttar Pradesh, ${ }^{46} 47$ the duration was substantially longer (around 12 months) among women $>30$ years compared with adolescents (3.5 months). Singh et al reported that in bivariate analysis, duration of amenorrhoea appeared longer in the two categories of adolescents $(<15$ and $15-20$ at time of marriage) compared with older women $(\mathrm{p}<0.01)$, but this association was no longer significant in multivariate analysis. ${ }^{51}$ Haggerty and Rutstein, in their analysis of 37 countries in the early 1990s, report a wide variability in duration of amenorrhoea. ${ }^{52}$ Sub-Saharan African countries had the longest durations of amenorrhoea; 14 of 18 countries had medians longer than a year. ${ }^{52}$ The four countries included in analyses for the Near East/North Africa region had the shortest durations, ranging from 4 to 6 months. ${ }^{52}$ The largest variability was documented in the Latin America/Caribbean region where median durations ranged from 4 months to 11 months. In most countries, there was either a pattern where duration of amenorrhoea increased with each older age group or a U-shape pattern where duration was longer among adolescents and women 35 years and older compared with the age groups between age 20 and 34 at time of birth. ${ }^{52}$ Across the four regions examined, the increase in duration of postpartum amenorrhoea with older age group was most notable in Sub-Saharan Africa. ${ }^{52}$ The most recent data available across the five studies examining duration of lactational amenorrhoea were collected in 2009 in India. ${ }^{51}$

\section{Theme 2: use of lactational amenorrhoea as a family planning method}

Four studies (from Bangladesh, Nigeria and Turkey) examined outcomes related to the use of lactational amenorrhoea as a family planning method among adolescents. Two of these studies included respondents who have never had a child together with those who have. ${ }^{4750}$ The first such study, ${ }^{47}$ assessing married adolescents from Bangladesh, found that $13.2 \%$ of adolescents who were not using contraceptives at the time of survey cited postpartum amenorrhoea as a reason for non-use. Audu and colleagues found that in their sample which included women with and without children in Nigeria, the percentage reporting ever-use of LAM was lowest among adolescents ( $5.0 \%$ among those 15-19 years), increasing to $10.0 \%$ among those 20-24 years and highest among women $35-39$ years old ( $p$ value of differences $<0.001) .^{50}$ One potential reason for the low percentage among the adolescent age group is that not all respondents in this sample have ever had children and therefore had the opportunity to have ever used LAM.

In a small sample of postpartum adolescents age $<20$ years $(n=10)$ in Turkey, Türk et al found that $70 \%$ considered themselves to be users of lactational amenorrhoea for family planning. ${ }^{49}$ This compared with $33 \%$ of those $20-29$ years old $(n=135)$ and $30 \%$ of those $\geq 30$ years old $(\mathrm{n}=43)$; $\mathrm{p}$ value $<0.0010 .{ }^{49}$ However, many who considered themselves users of LAM also reported having menses (28 of 64 self-reported users of LAM, not disaggregated by age group), and one-third of them became pregnant during the study follow-up period. ${ }^{49}$ Authors of this study highlighted that while LAM is one of the main family planning methods used in their sample, women might not be sufficiently aware of the criteria/ conditions for its effective use. The study on use of LAM as a family planning method with the most recent data was conducted in Turkey in $2010-2012 .{ }^{48}$ It found that $50.6 \%$ of those $10-19$ years old were using lactational amenorrhoea as a family planning method compared with $33 \%$ among women $20-35$ years. ${ }^{48}$ LAM was the most preferred method of contraception in this study (no quantitative indicators were provided to support this statement). ${ }^{48}$ Contraceptive failure in the adolescent age group was $2.37 \%$ in the first year post partum (12 unintended pregnancies among 506 adolescents in sample), compared with $2.0 \%$ in older age group. However, the failure rates were not available for LAM users separately. ${ }^{48}$

\section{Mechanisms for differences in lactational amenorrhoea between adolescents and older women}

Given the variability in findings on duration of amenorrhoea and the use of lactational amenorrhoea method between adolescents and older women in the included studies, we attempted to understand the potential mechanisms that study authors found or proposed. The main determinant of the length of lactational amenorrhoea is the duration and intensity of breast feeding. Few of the included studies attempted to interpret the findings relevant to adolescents or elucidate the mechanisms which might lead to differences in lactational amenorrhoea between adolescents and women older than 20 years, whether on a more granular or more distal levels. For 


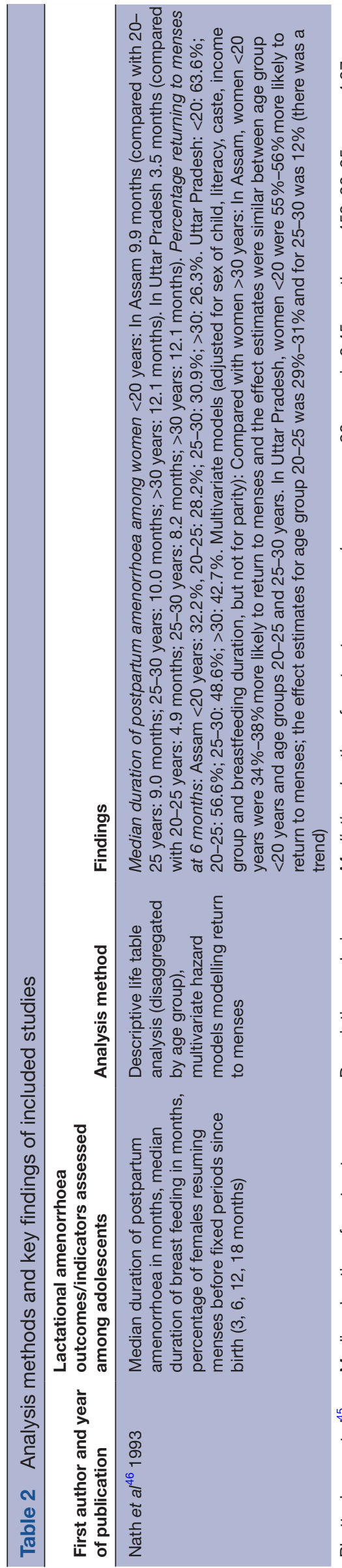

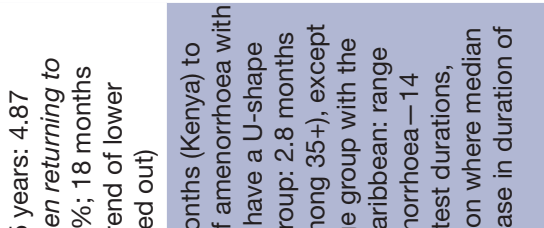

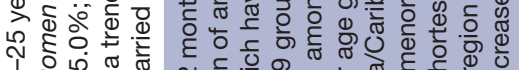
光

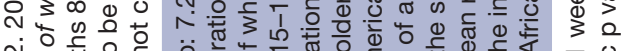

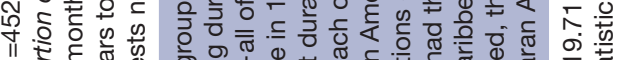
II

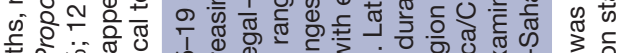

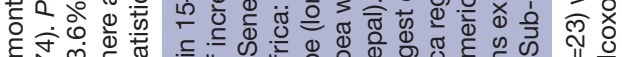

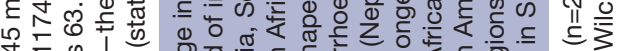

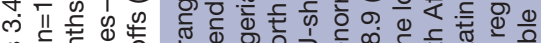

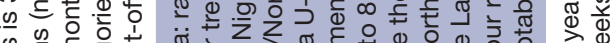

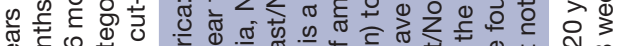
ब

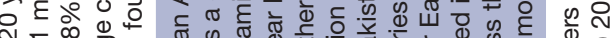
V.厂

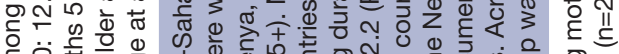

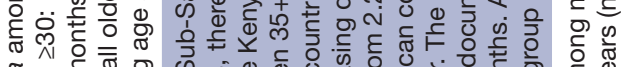

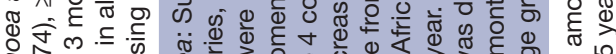

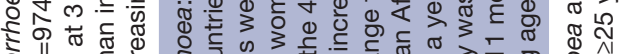

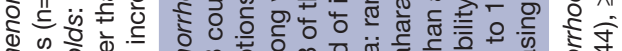

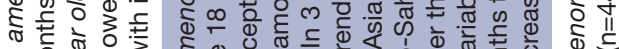

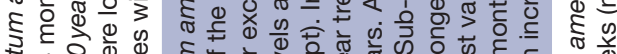

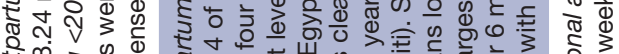

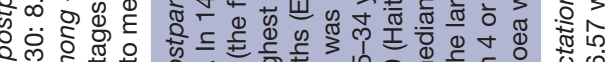

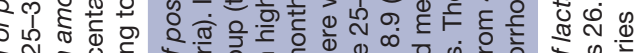

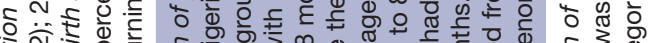

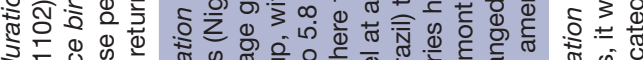

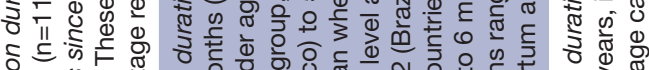

蛋

市

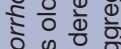

है

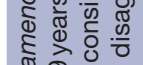

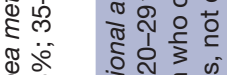

ले

敢

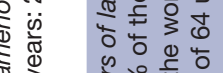

बं से

ఖ

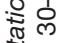

क्ष

प्ष

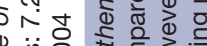

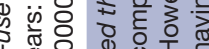

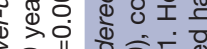

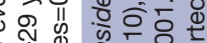

논

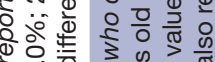

c.

就市

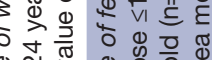

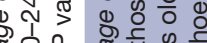

刃ㅇ. ㅁ.

ذِّ

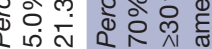

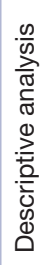

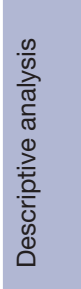

葛

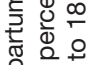

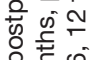

능 ᄃ $\leq$ 茂

苛

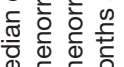

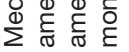

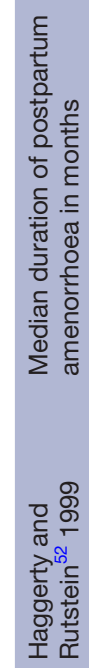

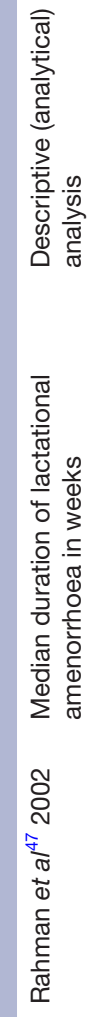

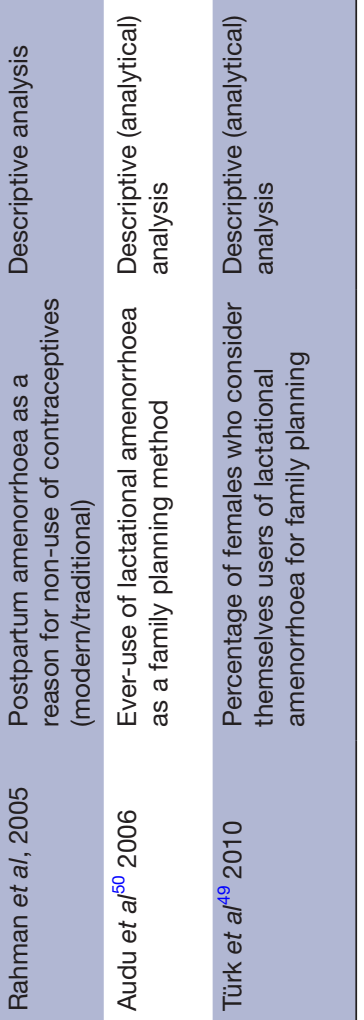




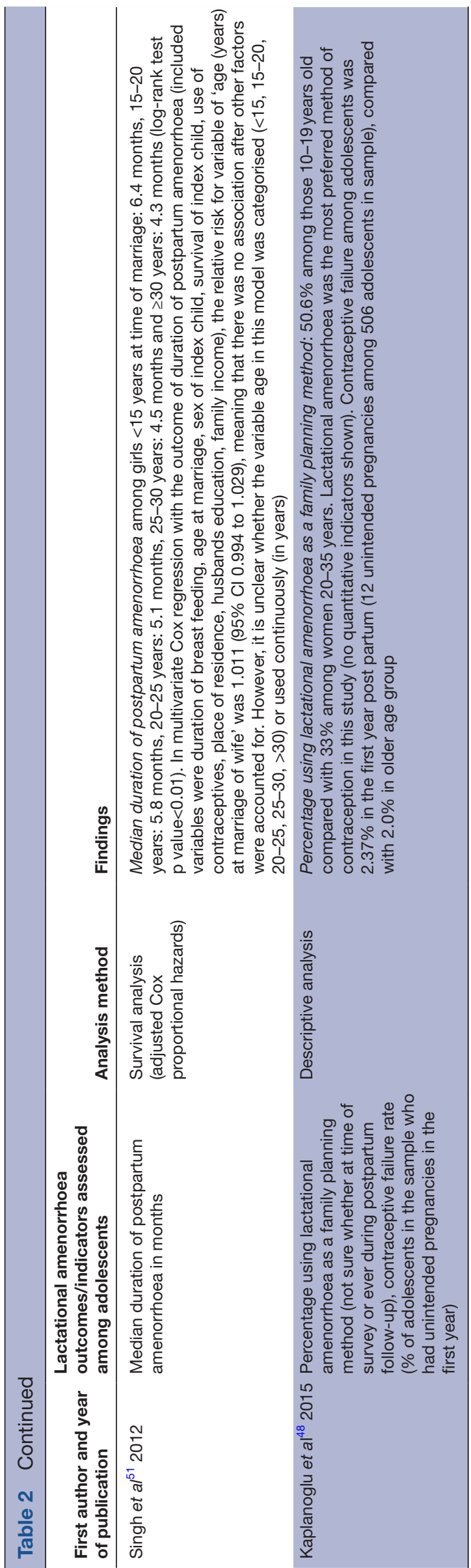

example, are any differences identified due to duration of breast feeding, suckling frequency (including nighttime feeding), timing and pattern of supplementation (including formula feeding), nutritional profile of the mother, or potentially an artefact of self-reporting by adolescents, or older women; or variations in knowledge and purposeful use of lactational amenorrhoea as a family planning method (potentially affected by education, literacy, and/or parity), or empowerment levels (ability to negotiate breastfeeding duration/frequency with other household members)?

Nath $e t a l,{ }^{46}$ who found large differences between the duration of lactational amenorrhoea between adolescents and older women in Uttar Pradesh but no differences in Assam, recognised that without detailed data on suckling pattern and supplementation, it is difficult to understand causes for the differences in duration of lactational amenorrhoea. Mechanisms they listed include different patterns of night nursing and nutritional status of women (malnourished women produce less breast milk which is also less nutritious, therefore their babies suckle longer, meaning women are likely to remain amenorrhoeic for longer periods). They explain the difference in duration of lactational amenorrhoea by age they found in Uttar Pradesh as being due to a biological delay in hormonal mechanisms responsible for ovulation (no more detail). Bhattacharya $e t a l,{ }^{45}$ who analysed the same data as Nath et al from Uttar Pradesh, mention the maternal nutrition mechanism to explain differences in duration of lactational amenorrhoea found between social groups and castes. If this mechanism were to be involved in the differences by age, then we would expect older women to be more malnourished as their lactational amenorrhoea duration is longer. Neither of the two studies using data from Uttar Pradesh tested this hypothesis. Lastly, Rahman et $a l,{ }^{47}$ who found an inverse U-shape pattern in duration of lactational amenorrhoea across age groups, offered no explanation or potential mechanisms for this association. However, they note that women in their sample did not always know their exact ages.

\section{Gaps in the available literature}

This review identified a scarcity of studies in LMICs focusing on lactational amenorrhoea among adolescent mothers, or more broadly, comparing lactational amenorrhoea characteristics and determinants across maternal age groups. The only comparative study across countries used data collected between 1990 and $1996,{ }^{52}$ and only one of the nine included studies used data collected in the past decade (since 2000) ${ }^{48}$ Geographically, we found gaps in available literature in several world regions, including Middle East/North Africa, where the comparative study reported very short durations of lactational amenorrhoea, and studies conducted in Latin America/Caribbean. ${ }^{52}$ Further, the studies using primary data included only married adolescents, meaning we have essentially no recent evidence about 
lactational amenorrhoea among unmarried adolescents. Last, none of the included studies used an intervention study design.

In regard to thematic areas, we did not find any studies assessing adolescents' knowledge of LAM as a postpartum family planning method. The study by Kaplanoglu et $a l^{48}$ from Turkey suggested there is insufficient knowledge among adolescents, but no formal assessment was conducted. Likewise, little is known about the effectiveness of LAM among adolescents using sufficiently large samples and follow-up time. In many of the included studies, sample sizes of adolescents were limited, and analysis methods restricted to descriptive or bivariate. Further analyses attempting to understand the factors associated with lactational amenorrhoea duration using more sophisticated analyses (multivariate adjusted models) are also needed. Crucially, more work is needed to understand the mechanisms leading to different durations of lactational amenorrhoea overall and the use of LAM as a family planning method across women's age groups. We found no qualitative research on lactational amenorrhoea among adolescents. Research on perceived enablers and barriers of breast feeding and lactational amenorrhoea, as well as on the reliability and validity of adolescents' report duration of lactational amenorrhoea are lacking.

We did not identify any studies assessing the role of postpartum or lactational amenorrhoea within the framework of proximate determinants of fertility ${ }^{53}$ among adolescents in LMICs. Such approach would need to incorporate a broader understanding of lactational amenorrhoea within a postpartum infecundability period, which combined lactational amenorrhoea with postpartum abstinence. Last, we did not find any studies on double (redundant) use of lactational amenorrhoea and other modern methods, ${ }^{35}$ or on the characteristics of transitions from the LAM to other contraceptives after 6 months post partum. The importance of postpartum use of modern methods appears key given the finding from Turkey that a high percentage of respondents incorrectly believed they were being protected from pregnancy by lactational amenorrhoea despite their menstrual period having returned. ${ }^{49}$

\section{DISCUSSION}

This scoping review systematically identified and summarised the findings of studies on lactational amenorrhoea among adolescent girls in LMICs. Using a set of selection criteria, two independent reviewers screened 75 full-text research papers published in the past 30 years and included a total of nine studies. Among these, only two had a main objective related to adolescents. The main reason for exclusion was a lack of disaggregation of individuals under study in the age categories 10-19 or 15-19 years old. Furthermore, several of the included studies which included adolescents and disaggregated lactational amenorrhoea estimates within this age group had very small sample sizes. There was heterogeneity in the findings about the duration of lactational amenorrhoea among adolescents compared with women $>20$ years. We also identified several important thematic gaps in currently available evidence, including adolescents' knowledge of LAM and transitions from the use of LAM to other contraceptive methods.

The heterogeneity in findings about duration of lactational amenorrhoea among adolescents compared with older women is not surprising given differences in breastfeeding practices across countries and contexts, as documented by Haggerty and Rutstein. ${ }^{52}$ Potential mechanisms leading to differences in duration of lactational amenorrhoea between adolescents and women older than 20 years were mentioned in two of the included studies. The authors highlighted the role of low maternal nutritional status (babies suckling more often and longer due to less nutritious milk) and biological delays in hormonal mechanisms responsible for ovulation. These mechanisms imply that adolescent mothers might have longer durations of postpartum amenorrhoea, which was not the case in all the settings from which studies included in this scoping review reported on this indicator. Weaning and supplementation patterns have an important effect on resumption of menses, as does complete cessation of breast feeding (eg, due to death of infant or transition to formula feeding among women with HIV).

Socioeconomic factors appear to have bi-directional effects on breast feeding: maternal education (higher education being linked to better awareness of benefits of breast feeding, including its contraceptive effects, and more receptive to health promotion), maternal occupation (competing demands on woman's time and higher likelihood of supplementation/formula feeding) and urbanisation/household wealth are linked to better ability to access and afford formula. ${ }^{54}$ Therefore, adolescents might have lower education levels, especially if they dropped out of education due to pregnancy and childrearing, and they might be more likely to supplement or wean earlier if they are returning back to school after childbirth. The effect of lower wealth among adolescent mothers compared with older mothers, ${ }^{55-58}$ particularly availability of disposable income to purchase formula and other items necessary to formula feed, might contribute to higher rates and duration of breast feeding among adolescents. Examination of the effect of marital status on lactational amenorrhoea in general, and understanding of lactational amenorrhoea among unmarried adolescents in particular, is completely absent from the identified literature. One might hypothesise that unmarried adolescent mothers who reside with their own family receive different types of support and advice with breast feeding and child-rearing compared with those living with husband or in-laws. However, how this and other factors affect breast feeding and lactational amenorrhoea is not known.

The effect of parity is crucial to the topic of this paper, as adolescent mothers have, on average, substantially 
lower parity compared with women $>20$ years of age. The effect of parity on duration of lactational amenorrhoea might also operate in both directions due to a combination of a cohort effect (older/higher parity women might breast feed longer) and mechanisms influencing breastfeeding patterns (nutritional depletion and/or lack of time to breast feed among high parity women). ${ }^{54}$ Adolescent mothers, of which a higher proportion are primiparous, might be more likely to encounter difficulties with initiating and sustaining breast feeding (eg, poor latch, engorgement, painful nipples), which in turn could make them more likely to supplement or wean early. ${ }^{59}$ Other issues linking low parity and breastfeeding behaviour among women in LMICs include, for example, higher rates of cesarean section ${ }^{60}$ and delivery in health facilities. ${ }^{61}$

It is possible that adolescent mothers have lower levels of knowledge about the existence and criteria for effective use of LAM compared with older women. This would likely be a combined effect of several factors, for example, lower education levels and lower parity (lack of previous use of reproductive/maternal health services where counselling on LAM use is covered) among adolescents. Lack of knowledge or inaccurate information about lactational amenorrhoea in general and LAM in particular can lead to two scenarios: (1) women are protected from conception by lactational amenorrhoea but are not using it intentionally as a contraceptive method (eg, due to lack of trust in it or knowledge about contraceptive effectiveness)-this might include women who also use another contraceptive method, and (2) women believe and report that they are using LAM, but are not doing so correctly, as the Turkish study found. ${ }^{49}$ Therefore, understanding the extent to which adolescents who report they are using LAM fulfil the criteria for this method is critical, and could be assessed through existing secondary data such as Demographic and Health Surveys. The low accuracy of women's self-report on the use of LAM for family planning was shown previously (26\% of reported users meet the criteria for correct LAM practice in analysis of data collected between 1998 and 2011 in 45 countries) ${ }^{62}$ However, we found no studies assessing whether the levels of reporting accuracy are differential between adolescent and older mothers, and if so, what are the patterns and mechanisms leading to such differentials.

We found no qualitative research on lactational amenorrhoea among adolescents. Research on perceived enablers and barriers of LAM use, as well as on the reliability and validity of adolescents' report of the duration of lactational amenorrhoea, are lacking. Levels of unmet need for family planning during the first year post partum are high in LMICs, ${ }^{63-65}$ and in some settings are higher among adolescent mothers compared with older women. ${ }^{66}$ No matter how effective the use of LAM is, it must be followed by another contraceptive method when one of the three criteria is no longer met and the woman desires to prevent a pregnancy. Evidence from LMICs shows that there is a gap in evidence on this transition among adolescents. ${ }^{67-69}$ It would be important to describe and address the context-specific barriers adolescent mothers are facing in choosing another modern method. This is particularly important because the range of family planning methods in the postpartum period, as well as the range offered/available to adolescents, might already be limited, and discontinuation levels of short-term contraceptives such as pills and injections are high. ${ }^{70-72}$ These barriers underscore the importance of health worker training and provision of counselling and support for breast feeding, ${ }^{71}$ with strong adolescent-friendly components.

Studies identified by this review were conducted mainly in four countries (India, Bangladesh, Turkey, Nigeria), while a cross-national study included 37 countries across Africa, Asia and Latin America. The four main countries have specific contexts in terms of sexual, reproductive and adolescent health, some of which might be similar to other LMIC settings, but may also may differ in terms of cultural and religious aspects. In addition, only one of these articles analysed data collected in the past 10 years, while national and international policies in terms of sexual and reproductive health and rights as well as maternal health service provision and utilisation has evolved. The limited number of settings and lack of recent data, in addition to the limited evidence base and the variable quality of measurement methods used, preclude us from making any broad generalisations.

\section{Limitations}

This scoping review has several limitations. First, we only conducted searches for references in English; relevant studies in other languages might have been excluded. This is particularly the case in regard to research describing high-fertility settings in French-speaking West Africa. Second, given our primary interest in adolescents, we might have missed studies which used maternal age or age groups in descriptive or multivariable analyses (eg, as a population characteristic or a confounder), but did not mention this in the title or abstract, and thus were not identified in title and abstract screening. However, we also reviewed reference lists of all included studies, which provided another opportunity to find such studies. Third, we only reviewed literature on adolescents from LMICs. Fourth, while the duration of postpartum insusceptibility is a result of whichever is longer, postpartum amenorrhoea or postpartum abstinence ${ }^{73}$; our focus was solely on the former in this review.

\section{CONCLUSION}

While lactational amenorrhoea is not relevant for prevention or delay of first adolescent childbirth, it might be important for lowering of repeat births among adolescents and young women, particularly those preceded by a short interpregnancy interval. Therefore, there is a need for more studies on duration of lactational amenorrhoea, knowledge and effective use of LAM for family planning 
among adolescents in a wide range of LMIC settings. Related to this, this study highlights the need for a better understanding of breastfeeding context-specific practices, barriers and enablers of lactational amenorrhoea use among adolescents, and transitioning from LAM onto other modern methods.

\section{Twitter Lenka Benova @lenkabenova}

Contributors MNSF and LB conceptualised the study, with input from TD and SB. Data were collected, screened and extracted by MNSF and LB. All coauthors contributed to the interpretation of findings. MNSF, LB and SB wrote the first draft of the manuscript, and all coauthors revised it critically.

Funding This study was funded by Research Foundation - Flanders (Senior Postdoctoral Fellowship).

\section{Competing interests None declared.}

Patient and public involvement Patients and/or the public were not involved in the design, or conduct, or reporting, or dissemination plans of this research.

\section{Patient consent for publication Not required.}

Provenance and peer review Not commissioned; externally peer reviewed.

Data availability statement All data relevant to the study are included in the article or uploaded as online supplemental information. All data relevant to the included studies are available in the manuscript text, tables and references.

Open access This is an open access article distributed in accordance with the Creative Commons Attribution Non Commercial (CC BY-NC 4.0) license, which permits others to distribute, remix, adapt, build upon this work non-commercially, and license their derivative works on different terms, provided the original work is properly cited, appropriate credit is given, any changes made indicated, and the use is non-commercial. See: http://creativecommons.org/licenses/by-nc/4.0/.

\section{REFERENCES}

1 Blum RW, Gates WH. Girlhood, not motherhood: preventing adolescent pregnancy. United Nations Population Fund (UNFPA), 2015.

2 Kuruvilla S, Bustreo F, Kuo T, et al. The global strategy for women's, children's and adolescents' health (2016-2030): a roadmap based on evidence and country experience. Bull World Health Organ 2016:94:398-400.

3 Neal S, Matthews Z, Frost M, et al. Childbearing in adolescents aged 12-15 years in low resource countries: a neglected issue. New estimates from demographic and household surveys in 42 countries. Acta Obstet Gynecol Scand 2012;91:1114-8.

4 United Nations, Department of Economic and Social Affairs, Population Division. World population prospects 2019, 2019 Available: https://population.un.org/wpp/ [Accessed 9 Jun 2020].

5 Loaiza E, Liang M. Adolescent pregnancy: a review of the evidence. New York: UNFPA, 2013.

6 United Nations, Department of Economic and Social Affairs, Population Division. Estimates and projections of family planning indicators 2020. New York: United Nations, 2020.

7 Chandra-Mouli V, McCarraher DR, Phillips SJ, et al. Contraception for adolescents in low and middle income countries: needs, barriers, and access. Reprod Health 2014;11:1.

8 Solo J, Festin M. Provider bias in family planning services: a review of its meaning and manifestations. Glob Health Sci Pract 2019;7:371-85.

9 Bankole A, Malarcher S. Removing barriers to adolescents access to contraceptive information and services. Stud Fam Plann 2010;41:117-24.

10 Nalwadda G, Mirembe F, Tumwesigye NM, et al. Constraints and prospects for contraceptive service provision to young people in Uganda: providers' perspectives. BMC Health Serv Res 2011;11:220.

11 Darroch JE, Woog V, Bankole A, et al. Costs and benefits of meeting the contraceptive needs of adolescents. Guttmacher Institute, 2016.

12 Williamson NE. Motherhood in childhood: facing the challenge of adolescent pregnancy. United Nations Population Fund, 2013.

13 ICF. The DHS program STATcompiler. funded by the USAID. Available: http://www.statcompiler.com [Accessed 13 Jun 2020].

14 Bellizzi S, Palestra F, Pichierri G. Adolescent women with unintended pregnancy in low- and middle-income countries: reasons for discontinuation of contraception. J Pediatr Adolesc Gynecol 2020;33:144-8.

15 World Health Organization. Global health estimates 2015: deaths by cause, age, sex, by country and by region, 2000-2015. Geneva: World Health Organization, 2016.

16 World Health Organization. Trends in maternal mortality: 1990 to 2015: estimates by WHO, UNICEF. UNFPA, World Bank Group and the United Nations Population Division Geneva, 2015.

17 Filippi V, Chou D, Ronsmans C, et al. Levels and causes of maternal mortality and morbidity, 2016.

18 Martin JA, Hamilton BE, Ventura SJ, et al. Births: final data for 2010. National vital statistics reports, 2012: 61.

19 Cleland J, Conde-Agudelo A, Peterson H, et al. Contraception and health. Lancet 2012;380:149-56.

20 Nortman D. Parental age as a factor in pregnancy outcome and child development. Reports on Population/Family Planning, 1974.

21 Benova L, Neal S, Radovich EG, et al. Using three indicators to understand the parity-specific contribution of adolescent childbearing to all births. BMJ Glob Health 2018;3:e001059.

22 Amongin D, Nakimuli A, Hanson C, et al. Time trends in and factors associated with repeat adolescent birth in Uganda: analysis of six demographic and health surveys. PLoS One 2020;15:e0231557.

23 Maravilla JC, Betts KS, Couto E Cruz C, Cruz CC, et al. Factors influencing repeated teenage pregnancy: a review and metaanalysis. Am J Obstet Gynecol 2017;217:e531:527-45.

24 Ahrens KA, Nelson H, Stidd RL, et al. Short interpregnancy intervals and adverse perinatal outcomes in high-resource settings: an updated systematic review. Paediatr Perinat Epidemiol 2019;33:O25-47.

25 Swaminathan A, Fell DB, Regan A, et al. Association between interpregnancy interval and subsequent stillbirth in 58 lowincome and middle-income countries: a retrospective analysis using demographic and health surveys. Lancet Glob Health 2020;8:e113-22.

26 Smith GC, Pell JP. Teenage pregnancy and risk of adverse perinatal outcomes associated with first and second births: population based retrospective cohort study. BMJ 2001;323:476-9.

27 Nerlander LM, Callaghan WM, Smith RA, et al. Short interpregnancy interval associated with preterm birth in US adolescents. Matern Child Health J 2015;19:850-8.

28 Cleland J, Bernstein S, Ezeh A, et al. Family planning: the unfinished agenda. Lancet 2006;368:1810-27.

29 Sridhar A, Salcedo J. Optimizing maternal and neonatal outcomes with postpartum contraception: impact on breastfeeding and birth spacing. Matern Health Neonatol Perinatol 2017;3:1.

30 Innocenti Publications. Innocenti Declaration 2005 on infant and young child feeding; 2007

31 McNeilly AS, Tay CC, Glasier A. Physiological mechanisms underlying lactational amenorrhea. Ann N Y Acad Sci 1994;709:145-55.

32 Van der Wijden C, Manion C. Lactational amenorrhoea method for family planning. Cochrane Database Syst Rev 2015:CD001329.

33 Short RV, Lewis PR, Renfree MB, et al. Contraceptive effects of extended lactational amenorrhoea: beyond the Bellagio Consensus. Lancet 1991;337:715-7.

34 Kennedy KI, Visness CM, Visness CM. Contraceptive efficacy of lactational amenorrhoea. Lancet 1992;339:76.

35 Cleland J, Shah IH, Benova L. A fresh look at the level of unmet need for family planning in the postpartum period, its causes and program implications. Int Perspect Sex Reprod Health 2015;41:155-62.

36 US AID: MCHIP. Lactational amenorrhea method, 2020. Available: https://www.mchip.net/interventions/family-planning/lactationalamenorrhea-method/

37 Festin MPR, Kiarie J, Solo J, et al. Moving towards the goals of FP2020 - classifying contraceptives. Contraception 2016;94:289-94.

38 Arksey H, O'Malley L. Scoping studies: towards a methodological framework. Int J Soc Res Methodol 2005;8:19-32.

39 Tricco AC, Lillie E, Zarin W, et al. PRISMA extension for scoping reviews (PRISMA-ScR): checklist and explanation. Ann Intern Med 2018;169:467-73.

40 The DHS pogram. Demographic and health surveys, 2019. Available: https://dhsprogram.com/search/

41 Population Council, 2019. Available: https://www.popcouncil.org/

42 WHO reproductive health library, 2019. Available: https://extranet. who.int/rhl/

43 Kennedy KI, Rivera R, McNeilly AS. Consensus statement on the use of breastfeeding as a family planning method. Contraception 1989;39:477-96.

44 The World Bank. World Bank Country Classification Washington: World Bank, 2019. Available: https://datahelpdesk.worldbank.org/ knowledgebase/topics/19280-country-classification 
45 Bhattacharya B, Singh KK, Singh U. Proximate determinants of fertility in Eastern Uttar Pradesh. Hum Biol 1995;67:867-86.

46 Nath DC, Singh KK, Land KC, et al. Breastfeeding and postpartum amenorrhea in a traditional society: a hazards model analysis. Soc Biol 1993;40:74-86.

47 Rahman M, Mascie-Taylor CGN, Rosetta L. The duration of lactational amenorrhoea in urban Bangladeshi women. J Biosoc Sci 2002;34:75-89.

48 Kaplanoglu M, Kaplanoglu D, Usman MG. Postpartum contraception in adolescents: data from a single tertiary clinic in southeast of Turkey. Glob J Health Sci 2015;7:80.

49 Türk R, Terzioğlu F, Eroğlu K. The use of lactational amenorrhea as a method of family planning in eastern Turkey and influential factors. $J$ Midwifery Womens Health 2010;55:e1-7.

50 Audu BM, Yahya SJ, Bassi A, Knowledge BA. Knowledge, attitude and practice of natural family planning methods in a population with poor utilisation of modern contraceptives. J Obstet Gynaecol 2006;26:555-60.

51 Singh NS, Singh NS, Narendra RK. Postpartum amenorrhoea among Manipuri women: a survival analysis. J Health Popul Nutr 2012;30:93.

52 Haggerty PA, Rutstein SO. Breastfeeding and complementary infant feeding, and the postpartum effects of breastfeeding. DHS Comparative Studies, 1999.

53 Bongaarts J. A framework for analyzing the proximate determinants of fertility. Popul Dev Rev 1978;4:105-32.

54 Berhanu B, Hogan D. Postpartum amenorrhoea in Ethiopia: the role of weaning, child death, and socioeconomic factors. Soc Biol 1998:45:80-95.

55 Rani M, Lule E. Exploring the socioeconomic dimension of adolescent reproductive health: a multicountry analysis. Int Fam Plan Perspect 2004;30:110-7.

56 Poudel S, Upadhaya N, Khatri RB, et al. Trends and factors associated with pregnancies among adolescent women in Nepal: pooled analysis of Nepal demographic and health surveys (2006, 2011 and 2016). PLoS One 2018;13:e0202107.

57 Chirwa GC, Mazalale J, Likupe G, et al. An evolution of socioeconomic related inequality in teenage pregnancy and childbearing in Malawi. PLoS One 2019;14:e0225374.

58 Castro R, Fajnzylber E. Income inequality and adolescent fertility in low-income countries. Cad Saude Publica 2017;33:e00203615.
59 Goyal RC, Banginwar AS, Ziyo F, et al. Breastfeeding practices: positioning, attachment (latch-on) and effective suckling - a hospitalbased study in Libya. J Family Community Med 2011;18:74.

60 Pereira CRVR, Fonseca VdeM, Couto de Oliveira MI, Oliveira M, et al. Assessment of factors that interfere on breastfeeding within the first hour of life. Rev Bras Epidemiol 2013;16:525-34.

61 Bergamaschi N, Oakley L, Benova L. Is childbirth location associated with higher rates of favourable early breastfeeding practices in SubSaharan Africa? J Glob Health 2019;9:010417.

62 Fabic MS, Choi Y. Assessing the quality of data regarding use of the lactational amenorrhea method. Stud Fam Plann 2013;44:205-21.

63 Ross JA, Winfrey WL. Contraceptive use, intention to use and unmet need during the extended postpartum period. Int Fam Plan Perspect 2001;27:20-7.

64 World Health Organization. Programming strategies for postpartum family planning; 2013.

65 Track 20. Trends in the uptake of postpartum family planning, 2017 Available: http://www.track20.org/pages/data analysis/in depth/ PPFP/trends.php [Accessed 12 Feb 2020].

66 Pasha O, Goudar SS, Patel A, et al. Postpartum contraceptive use and unmet need for family planning in five low-income countries. Reprod Health 2015;12 Suppl 2:S11.

67 Mumah JN, Machiyama K, Mutua M, et al. Contraceptive adoption, discontinuation, and switching among postpartum women in Nairobi's urban slums. Stud Fam Plann 2015;46:369-86.

68 Rossier C, Bradley SEK, Ross J, et al. Reassessing unmet need for family planning in the postpartum period. Stud Fam Plann 2015;46:355-67.

69 Machiyama K, Cleland J. Insights into unmet need in Kenya. London: London School of Hygiene \& Tropical Medicine, 2013.

70 Ali M, Cleland J, Shah I. Causes and consequences of contraceptive discontinuation: evidence from 60 demographic and health surveys, 2012. World Health Organisation ISBN, 2013.

71 Ali MM, Cleland J. Oral contraceptive discontinuation and its aftermath in 19 developing countries. Contraception 2010;81:22-9.

72 Cavallaro FL, Duclos D, Cresswell JA, et al. Understanding 'missed appointments' for pills and injectables: a mixed methods study in Senegal. BMJ Glob Health 2018;3:e000975.

73 Brown M. When ancient meets modern: the relationship between postpartum non-susceptibility and contraception in Sub-Saharan Africa. J Biosoc Sci 2007;39:493-515. 